The Chinese Society of Theoretical and Applied Mechanics

Chinese Journal of Mechanics Press, Beijing, China

Allerton Press, INC., New York, U.S.A.

\title{
MECHANICS OF RELAXING SiGe ISLANDS ON A VISCOUS GLASS*
}

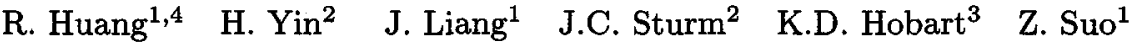 \\ ${ }^{1}$ (Mechanical and Aerospace Engineering Department and Princeton Materials Institute, \\ Princeton University, Princeton, NJ 08544, USA) \\ ${ }^{2}$ (Center for Photonics and Optoelectronic Materials and Department of Electrical Engineering, \\ Princeton University, Princeton, NJ 08544, USA) \\ ${ }^{3}$ (Naval Research Laboratory, Washington, DC 20375, USA) \\ ${ }^{4}$ (Department of Aerospace Engineering and Engineering Mechanics, University of Texas at \\ Austin, Austin, TX 78712, USA)
}

\begin{abstract}
A process has been developed recently to fabricate a structure comprising, from top to bottom, a SiGe thin film, a glass layer, and a $\mathrm{Si}$ wafer. The $\mathrm{SiGe}$ film is a perfect crystal, and is under biaxial compression. The SiGe film is patterned into islands. On annealing, the glass flows and the islands relax. The resulting strain-free islands are used as substrates, to grow epitaxial optoelectronic devices. This article describes a series of studies on the annealing process, combining experiment and theory. A small island relaxes by expansion, starting at the edges and diffusing to the center. A large island wrinkles before the expansion reaches the center. After some time, the wrinkles either disappear, or cause the island to fracture. We model the island as an elastic plate, and the glass layer as a viscous liquid. The strains in the islands are measured by X-ray diffraction and Raman spectroscopy, and the wrinkle amplitudes by atomic force microscope. The data are compared with the theoretical predictions. We determine the conditions under which the islands relax by expansion without significant wrinkling, and demonstrate that a cap layer suppresses wrinkles, relaxing a large island crack-free.
\end{abstract}

KEY WORDS: semiconductor fabrication, stress relaxation, viscous flow, elastic plate, fracture

\section{INTRODUCTION}

Micro and nano fabrication processes provide a meeting ground between theory and experiment, and between science and technology ${ }^{[1,2]}$. An extensively studied example involves the growth of an epitaxial semiconductor film on a semiconductor substrate ${ }^{[3,4]}$. Other fabrication processes, such as wafer bonding, nanoimprint, electrodeposition, and self-assembly, all pose fascinating mechanics problems ${ }^{[5 \sim 13]}$. This article focuses on a specific process to fabricate $\mathrm{SiGe}$ on a silicon wafer. The two elements, $\mathrm{Si}$ and $\mathrm{Ge}$, form solid solutions of any

Received 18 June 2002

* The project supported by NSF (CMS-9820713), DARPA (N66001-00-1-8957), ARO (DAA655-98-10270), and New Jersey. Science and Technology Commission 
proportion. Ge has a lattice constant $4 \%$ larger than that of Si. By changing the composition, SiGe solutions provide a range of lattice constants. On a relaxed $\mathrm{SiGe}$, an epitaxial $\mathrm{Si}$ film is in tension and has a high electron mobility ${ }^{[14]}$, and an epitaxial Ge film is in compression and has a high hole mobility ${ }^{[15]}$. The strained Si and Ge films are used for high-speed electronics ${ }^{[16]}$. Furthermore, Ge matches the lattice constant of GaAs, a compound semiconductor for lasers ${ }^{[17]}$. Consequently, by relaxing SiGe (including pure Ge) on silicon, one can integrate high-performance electronic and optoelectronic devices on a single wafer.

One way to relax a SiGe layer on a silicon wafer is to allow dislocations to accommodate the lattice mismatch between SiGe and Si. Unfortunately, in addition to such geometrically necessary dislocations (i.e., the misfit dislocations), statistically stored dislocations (i.e., the threading dislocations) are abundant. The latter will thread into the epitaxial devices grown above the SiGe layer, impairing the device performance. An improved approach is to grade the SiGe composition during growth ${ }^{[18,19]}$. The top SiGe layer grown this way has a lower threading dislocation density than a $\mathrm{SiGe}$ of the same composition grown directly on Si. The density, however, is still too high for some applications. Besides, this approach requires a thick total SiGe layer, which is a disadvantage.

Another way to relax $\mathrm{SiGe}$ is to use various "compliant substrates" [20 24]. Recently, Hobart and co-workers have studied the relaxation of SiGe islands transferred to a viscous borophosphorosilicate glass (BPSG) layer ${ }^{25]}$. Following this initial work, we and others have studied this process with a combination of experiment and theory ${ }^{[26 \sim 33]}$. This article integrates these studies, provides some evidences for wrinkle-induced fracture, and demonstrates an improved method to fabricate large, zero-defect, and strain-relaxed SiGe islands.

\section{FABRICATION PROCESS}

Figure 1 illustrates the fabrication process ${ }^{[25]}$. Grow an epitaxial $\mathrm{SiGe}$ film on a $\mathrm{Si}$ host wafer. The SiGe film has a larger lattice constant than that of $\mathrm{Si}$, and is under biaxial compression. To avert dislocations, the SiGe film is below the critical thickness. Separately coat a BPSG layer on a Si handle wafer. Stack the two wafers by the wafer bonding method, with the SiGe facing the BPSG. Remove the Si host wafer, and then pattern the SiGe film into islands. Unless otherwise noted, the BPSG layer is $200 \mathrm{~nm}$ thick, and the SiGe film is $30 \mathrm{~nm}$ thick. The experiment uses $\mathrm{Si}_{0.7} \mathrm{Ge}_{0.3}$, with lattice constant $1.2 \%$ larger than that of $\mathrm{Si}$, patterned into square islands of various sizes, $L=10 \mu \mathrm{m} \sim 500 \mu \mathrm{m}$. Before annealing, the SiGe islands are under the $1.2 \%$ biaxial compressive strain, because the island size is much larger than the island thickness, and the BPSG is solid at room temperature. On annealing above the glass transition temperature, the glass flows, and the islands relax elastically. The process involves no dislocations and, in principle, produces zero-defect, relaxed SiGe islands. The resulting strain-free islands can be used as substrates to grow epitaxial optoelectronic devices. For this purpose, large islands are desired.

Islands of various sizes are annealed above the glass transition temperature of BPSG ${ }^{[33]}$. Small islands $(L<30 \mu \mathrm{m})$ expand, but large islands wrinkle. After a long time of annealing, the wrinkles disappear for mid-sized islands $(30 \mu \mathrm{m}<L<80 \mu \mathrm{m})$, but cause fracture for large islands. Figure 2 shows a part of a $100 \mu \mathrm{m}$ island annealed for $90 \mathrm{~min}$ at $790^{\circ} \mathrm{C}$. At the corner, the island expands in both inplane directions, and remains flat. At each edge, the island expands in one direction, and wrinkles in the other. At the center, and island wrinkles in a complicated way. Figure 3 shows a $200 \mu \mathrm{m}$ island annealed for $90 \mathrm{~min}$ at $790^{\circ} \mathrm{C}$. 


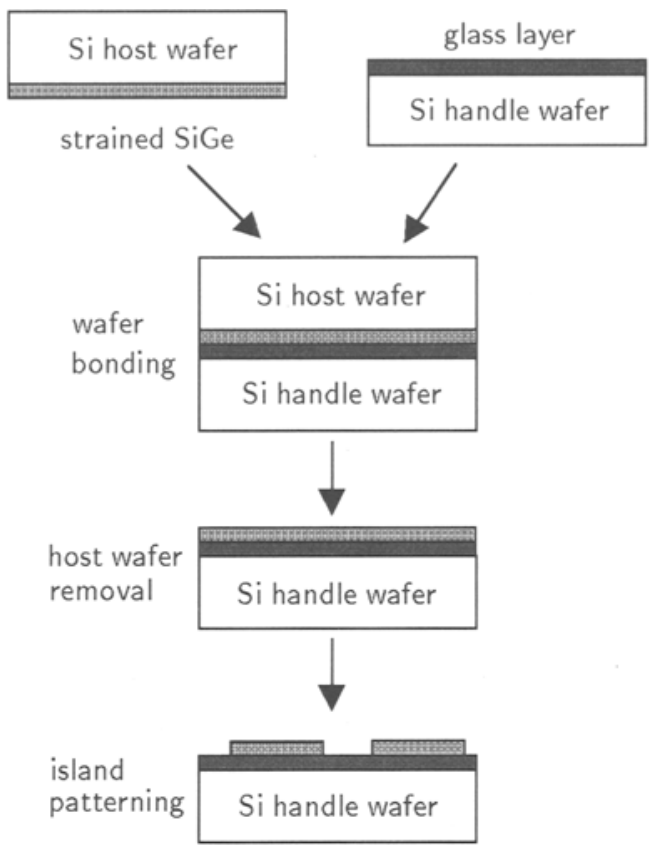

Fig.1 Schematic of the fabrication process

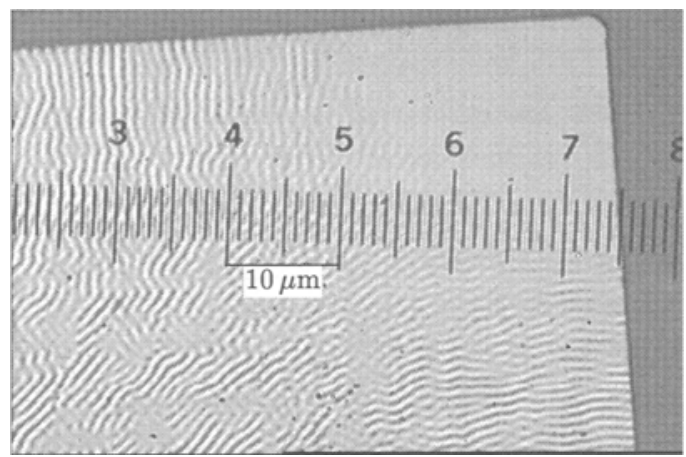

Fig.2 Part of a $100 \mu \mathrm{m}$ by $100 \mu \mathrm{m}$ island annealed for $90 \mathrm{~min}$ at $790^{\circ} \mathrm{C}^{[33]}$

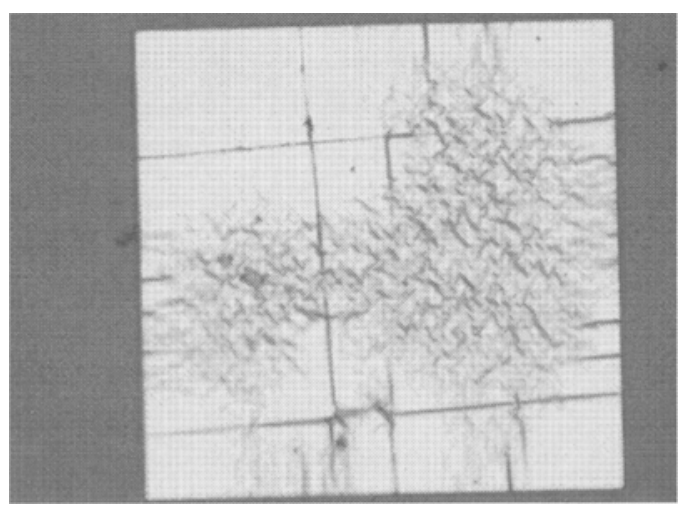

Fig.3 A $200 \mu \mathrm{m}$ by $200 \mu \mathrm{m}$ island annealed for $90 \mathrm{~min}$ at $790^{\circ} \mathrm{C}$ 
In addition to wrinkles, channel cracks form in the island, probably initiated from the island edges. It is the wrinkle-induced fracture that limits the size of the islands to be relaxed.

\section{GENERAL THEORY}

Figure 4 illustrates the model. The SiGe island is taken to be elastic, the BPSG viscous, and the Si wafer rigid. Maintain the continuity of velocities and tractions at the SiGe-BPSG interface, and zero velocity at the BPSG-Si interface. The island is flat and under biaxial stresses in the initial state, which serves as the reference state. On annealing, the island expands and wrinkles. Let $u_{1}$ and $u_{2}$ be the inplane displacements, and $w$ be the deflection, all set to zero in the initial state. On the SiGe-BPSG interface, let $q$ be the normal traction, and $T_{1}$ and $T_{2}$ be the shear tractions. The elasticity of the SiGe island relates the tractions on the interface to the displacement field. The viscous flow of the BPSG layer relates the velocity field to the tractions. The combination relates the velocity field to the displacement field, governing the system. The following is the theory.

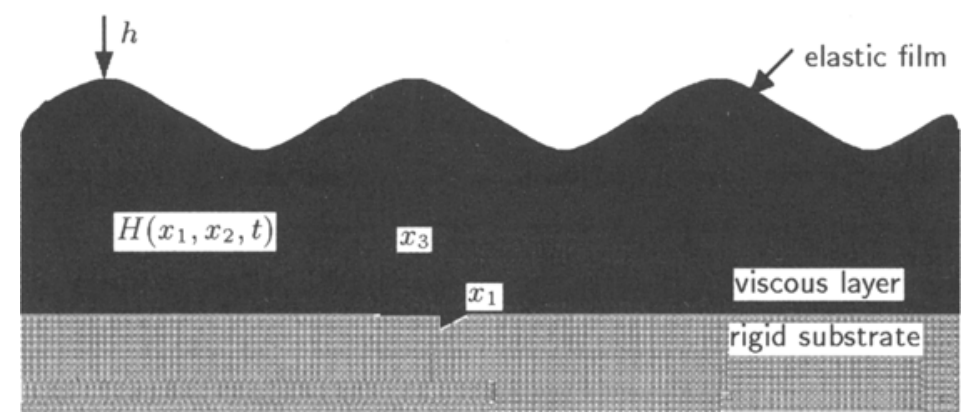

Fig.4 Schematic of the model

To describe both expansion and wrinkling, we model the elastic SiGe island using the von Kármán plate theory ${ }^{[34,35]}$. The membrane strains have three contributions

$$
\varepsilon_{\alpha \beta}=-\varepsilon_{0} \delta_{\alpha \beta}+\frac{1}{2}\left(\frac{\partial u_{\alpha}}{\partial x_{\beta}}+\frac{\partial u_{\beta}}{\partial x_{\alpha}}\right)+\frac{1}{2} \frac{\partial w}{\partial x_{\alpha}} \frac{\partial w}{\partial x_{\beta}}
$$

The first comes from the initial biaxial compressive strain, $\varepsilon_{0}$ being the magnitude; the second from the gradients of the inplane displacements; and the third from the large deflection. The Greek subscript takes values 1 and 2. Hooke's law relates the membrane forces $N_{\alpha \beta}$ to the membrane strains $\varepsilon_{\alpha \beta}$

$$
N_{\alpha \beta}=E h\left[\frac{1}{1+\nu} \varepsilon_{\alpha \beta}+\frac{\nu}{1-\nu^{2}} \varepsilon_{\gamma \gamma} \delta_{\alpha \beta}\right]
$$

where $E$ is Young's modulus, $\nu$ Poisson's ratio, and $h$ thickness of the island. A repeated Greek subscript implies summation over 1 and 2. The inplane force balance of a plate element requires that

$$
T_{\alpha}=\frac{\partial N_{\alpha \beta}}{\partial x_{\beta}}
$$

The out-of-plane force balance and the moment balance of a plate element require that

$$
q=-\frac{E h^{3}}{12\left(1-\nu^{2}\right)} \frac{\partial^{4} w}{\partial x_{\alpha}^{2} \partial x_{\beta}^{2}}+N_{\alpha \beta} \frac{\partial^{2} w}{\partial x_{\alpha} \partial x_{\beta}}+T_{\alpha} \frac{\partial w}{\partial x_{\alpha}}
$$


In essence, the plate theory provides an algorithm to calculate the tractions on the interface, $T_{1}, T_{2}, q$, in terms of the displacement field of the island, $u_{1}, u_{2}, w$.

We model the BPSG layer above the glass transition temperature as an incompressible viscous liquid, adopt the Stokes creep theory ${ }^{[36]}$ in this section, and describe specialized models in the subsequent sections. The stress field in the glass layer, $\sigma_{i j}$, is in equilibrium, so that

$$
\frac{\partial \sigma_{i j}}{\partial x_{j}}=0
$$

The Latin indices take values 1,2 and 3, and a repeated Latin index implies summation over 1,2 and 3 . The stresses relate to the velocities $V_{i}$ as

$$
\sigma_{i j}=-p \delta_{i j}+\eta\left(\frac{\partial V_{i}}{\partial x_{j}}+\frac{\partial V_{j}}{\partial x_{i}}\right)
$$

where $p$ is the pressure and $\eta$ the viscosity of the glass. Incompressibility requires that

$$
\frac{\partial V_{i}}{\partial x_{i}}=0
$$

The velocity field at the BPSG-Si interface vanishes, and the tractions on the BPSG-SiGe interface are $T_{1}, T_{2}, q$. Equations $(5) \sim(7)$, together with the boundary conditions, define a moving boundary value problem. In essence, the fluid mechanics of the BPSG layer provides an algorithm to calculate the velocity field in terms of the tractions on the BPSG-SiGe interface.

The coupled solid-liquid problem is nonlinear, with solutions of several kinds, corresponding to the growing wrinkles, the equilibrium wrinkles, the flat strained blanket film, and the relaxed island. The following sections reduce the general theory to simpler models, and compare the solutions to experiments.

\section{ISLAND EXPANSION}

The problem is greatly simplified if we focus on the island expansion, neglecting the wrinkles, and assuming the shear stress in the BPSG layer to be uniform across its thickness ${ }^{[26,27]}$. The resulting approximation, known as the shear lag model, dates back to the studies of tectonic plate drift ${ }^{[37 \sim 39]}$.

To see how the shear lag model describes the island expansion, here we examine the relaxation in the $x_{1}$ direction. The only nonzero displacement component is $u_{1}\left(x_{1}, t\right)$. From (1), the membrane strains are

$$
\varepsilon_{11}=-\varepsilon_{0}+\frac{\partial u_{1}}{\partial x_{1}} \quad \varepsilon_{22}=-\varepsilon_{0} \quad \varepsilon_{12}=0
$$

Hooke's law (2) gives the membrane force component in the $x_{1}$ direction

$$
N_{11}=-\frac{E h}{1-\nu} \varepsilon_{0}+\frac{E h}{1-\nu^{2}} \frac{\partial u_{1}}{\partial x_{1}}
$$

The first term is the initial compressive membrane force, and the second term accounts for the expansion. The inplane force balance in the $x_{1}$ direction, of a SiGe film element, relates the shear traction on the interface, $T_{1}$, to the divergence of the membrane force, namely

$$
\frac{\partial N_{11}}{\partial x_{1}}=T_{1}
$$


Assume that the shear stress in the BPSG is uniform across its thickness, so is the shear strain-rate, approximated by $\left(\partial u_{1} / \partial t\right) / H$, where $H$ is the thickness of the BPSG. The shear stress is linear in the shear strain-rate

$$
T_{1}=\frac{\eta}{H} \frac{\partial u_{1}}{\partial t}
$$

A combination of $(9) \sim(11)$ leads to

$$
\frac{\partial u_{1}}{\partial t}=\frac{E h H}{\left(1-\nu^{2}\right) \eta} \frac{\partial^{2} u_{1}}{\partial x_{1}^{2}}
$$

Equation (12) has the form of the diffusion equation, with $E h H /\left(1-\nu^{2}\right) \eta$ being the effective diffusivity. Now consider the expansion of an island of length $L$. At $t=0$, the membrane force $N_{11}$ vanishes at the two edges, and equals $-E h \varepsilon_{0} /(1-\nu)$ inside the island. On annealing, the expansion starts at the island edges, and diffuses to the island center. As $t \rightarrow \infty$, the membrane force $N_{11} \rightarrow 0$ in the entire island.

The solution of this classical initial-boundary value problem takes the form of an infinite series, the slowest decaying term being proportional to $\exp \left(-t / t_{\mathrm{E}}\right)$, with

$$
t_{\mathrm{E}}=\frac{1-\nu^{2}}{\pi^{2}} \frac{L^{2}}{H h} \frac{\eta}{E}
$$

We take $t_{\mathrm{E}}$ as the time scale for the island expansion. Equation (13) is also obtained by dimensional considerations, except for the factor $\pi^{2}$. The expansion time $t_{\mathrm{E}}$ depends on the dimensionless ratio $L^{2} / H h$, and the ratio $\eta / E$ that has the dimension of time. A large island takes a long time to relax.

The shear lag model has also been formulated in two dimensions ${ }^{[39]}$, and solved numerically for the expansion of a square island ${ }^{[27]}$. Figure 5 plots the strain scaled by the initial mismatch strain, against the time scaled by the square of the island size ${ }^{[33]}$. With such scaling, the shear lag model predicts one single curve in each plot for all island sizes. The experimental data for islands of various sizes fall on the same curve, confirming this scaling law. We use X-ray diffraction to measure the average strain in the island (Fig.5(a)), and Raman spectroscopy to measure the strain at the island center (Fig.5(b)). Comparing

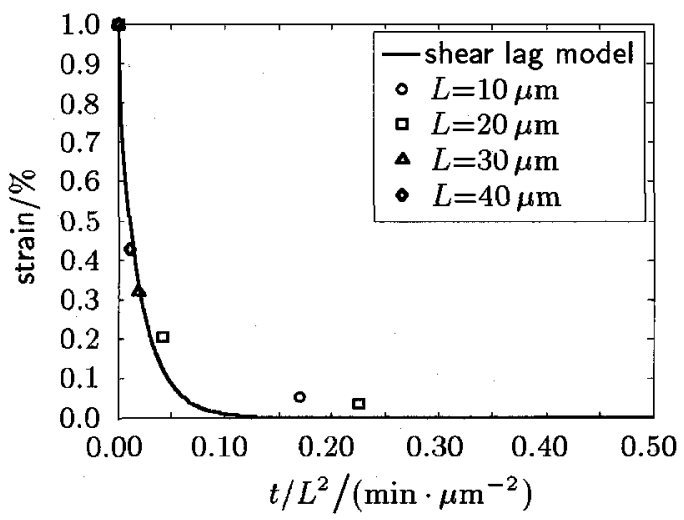

(a) The average strain during annealing at $790^{\circ} \mathrm{C}$

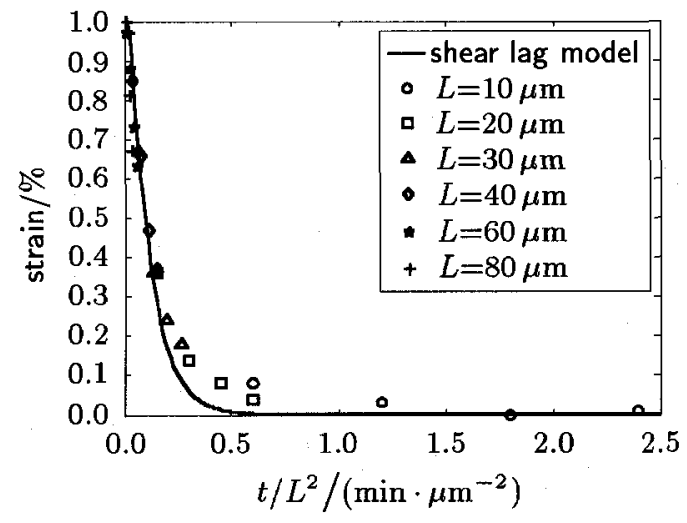

(b) The strain at the island center during annealing at $750^{\circ} \mathrm{C}$

Fig.5 Comparison between the shear lag model and the experiment data ${ }^{[33]}$ 
the experimental data and the numerical solution, we determine the viscosity of BPSG: $\eta=12.2 \mathrm{GPa} \cdot \mathrm{s}$ at $790^{\circ} \mathrm{C}$, and $\eta=52.5 \mathrm{GPa} \cdot \mathrm{s}$ at $750^{\circ} \mathrm{C}$.

\section{GROWING WRINKLES}

We next consider wrinkles in a blanket SiGe film (i.e., an infinite island). When a flat blanket film is under a uniform biaxial compression, generating no tractions on the SiGeBPSG interface, the BPSG does not creep. This equilibrium state is a trivial solution to the problem posed in Section 3. The equilibrium state, however, is unstable. The elastic energy stored in the SiGe film reduces when the film wrinkles. To describe the salient features of the wrinkle dynamics, here we give a simplified analysis, assuming that the BPSG layer is so thick that its thickness does not enter into the consideration. The analysis follows those in $[28,40]$. More comprehensive analyses are given in [29 31].

Perturb the film with a sinusoidal deflection

$$
w\left(x_{1}, t\right)=A(t) \sin \left(\frac{2 \pi x_{1}}{\lambda}\right)
$$

where $A(t)$ is the time-dependent wrinkle amplitude, and $\lambda$ the wrinkle wavelength. The analysis assumes that the amplitude $A$ is small, and keeps only the terms linear in $A$.

To the first order in $A$, the membrane force is unperturbed, given by its initial value $N_{11}=-E h \varepsilon_{0} /(1-\nu)$, and the shear traction $T_{1}$ vanishes. Equation (4) becomes

$$
q=\left[-\frac{E h^{3}}{12\left(1-\nu^{2}\right)}\left(\frac{2 \pi}{\lambda}\right)^{4}+\frac{E h \varepsilon_{0}}{1-\nu}\left(\frac{2 \pi}{\lambda}\right)^{2}\right] A(t) \sin \left(\frac{2 \pi x_{1}}{\lambda}\right)
$$

Subject to this traction on the interface, the BPSG creeps, and its velocity field is determined by solving the creep problem posed in Section 3. The velocity of the surface, $\partial w / \partial t$, is proportional to a representative strain rate, $q / \eta$, and to a length scale, $\lambda$, giving

$$
\frac{\partial w}{\partial t}=\frac{\lambda q}{4 \pi \eta}
$$

The factor $4 \pi$ is determined by solving the boundary value problem.

Inserting (14) and (15) into (16) yields a differential equation $\mathrm{d} A / \mathrm{d} t=s A$, with

$$
s=\frac{E}{24 \eta\left(1-\nu^{2}\right)}\left[-\left(\frac{2 \pi h}{\lambda}\right)^{3}+12(1+\nu) \varepsilon_{0}\left(\frac{2 \pi h}{\lambda}\right)\right]
$$

The solution to this differential equation is $A(t)=A_{0} \exp (s t)$, where $A_{0}$ is the initial wrinkle amplitude. The parameter $s$ governs the rate of change in the wrinkle amplitude. When a flat film wrinkles, the bending increases the energy stored in the film, but the inplane compression does work to decrease the energy. The two actions correspond to the two terms in the bracket of (17).

Figure 6 sketches $s$ as a function of the wavelength. The value of $s$ vanishes at the critical wavelength

$$
\lambda_{\mathrm{c}}=\frac{\pi h}{\sqrt{3(1+\nu) \varepsilon_{0}}}
$$




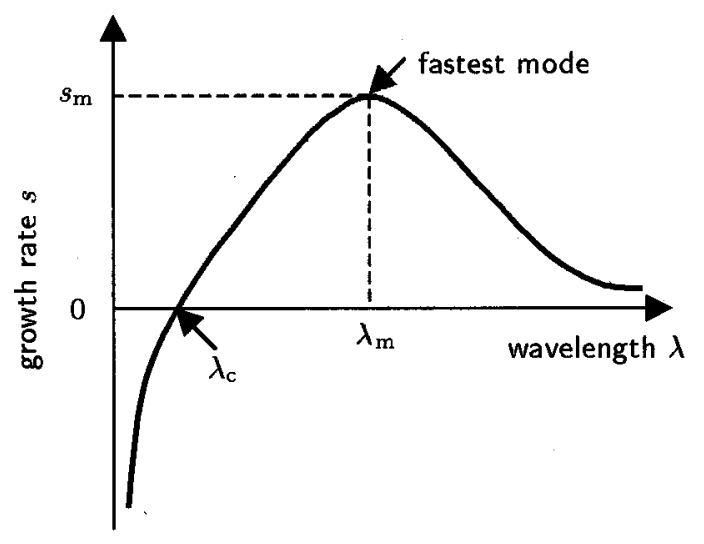

Fig.6 Schematic of the wrinkle growth rate as a function of the wrinkle wavelength

which reproduces the Euler buckling condition. When $\lambda<\lambda_{\mathbf{c}}$, the bending costs too much energy, $s<0$, and the wrinkles decay over time. When $\lambda>\lambda_{\mathbf{c}}$, the work done by the inplane compression prevails, $s>0$, and the wrinkles amplify over time. When $\lambda \rightarrow \infty$, creep over the long distance takes a long time, so that $s \rightarrow 0$.

The value of $s$ reaches a maximum value

$$
s_{\mathrm{m}}=\frac{2 \sqrt{1+\nu} \varepsilon_{0}^{3 / 2}}{3(1-\nu)} \frac{E}{\eta}
$$

at the wavelength

$$
\lambda_{\mathrm{m}}=\frac{\pi h}{\sqrt{(1+\nu) \varepsilon_{0}}}
$$

Wrinkles of this wavelength amplify the fastest. Using $\nu=0.3, \varepsilon_{0}=0.012$, and $h=30 \mathrm{~nm}$, Eq. (20) gives $\lambda_{\mathrm{m}}=0.75 \mu \mathrm{m}$. The wavelength observed experimentally is about $1 \mu \mathrm{m}$ (Fig.2). The agreement is reasonable.

The fastest amplifying wrinkles obey $A(t)=A_{0} \exp \left(s_{\mathrm{m}} t\right)$. The time for the wrinkles to amplify from the initial amplitude $A_{0}$ to amplitude $A$ is

$$
t_{\mathrm{W}}=\frac{3(1-\nu)}{2 \sqrt{1+\nu} \varepsilon_{0}^{3 / 2}} \ln \left(\frac{A}{A_{0}}\right) \frac{\eta}{E}
$$

We measure the surface roughness as a function of annealing time by using the atomic force microscope. Figure 7 plots the experimental data for $30 \mathrm{~nm}$ and $60 \mathrm{~nm}$ thick SiGe films ${ }^{[3]}$. The predictions of the linear perturbation analysis correspond to the straight lines in Fig.7. We use the viscosity extracted in the previous section from the inplane expansion of small islands, and use the initial amplitude $A_{0}$ as the single fitting parameter. The agreement between theory and experiment is excellent for the initial stage of wrinkling. 


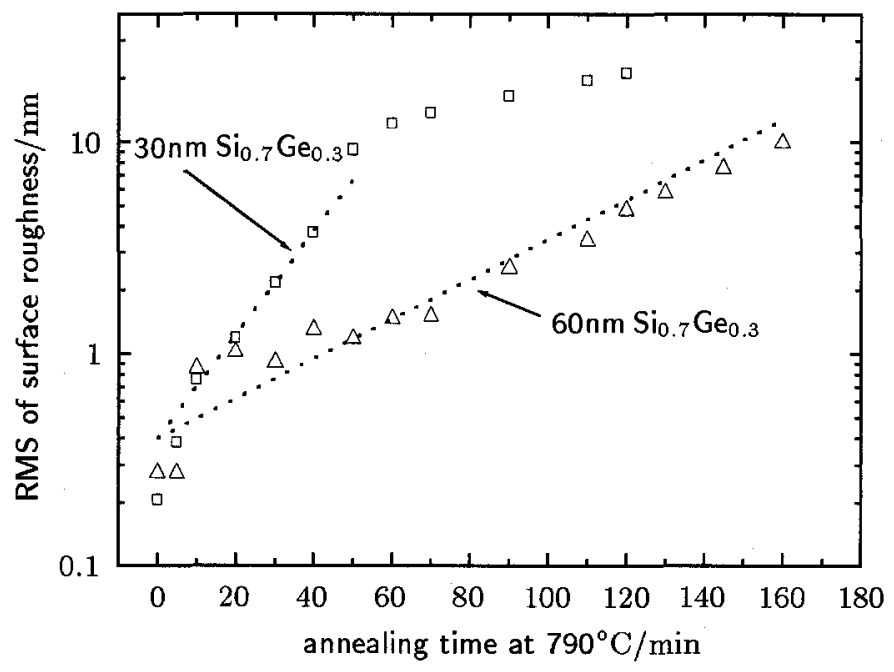

Fig.7 Surface roughness as a function of annealing time. The dotted lines are from the linear perturbation analysis and the open symbols are from experiments ${ }^{[33]}$

\section{EQUILIBRIUM WRINKLES}

Figure 7 shows that, for the $30 \mathrm{~nm}$ film, the wrinkle amplitude saturates after about $1 \mathrm{~h}$ annealing. That is, the wrinkles attain an equilibrium state, the BPSG stops creeping, and the tractions on the interface vanish. Such an equilibrium state is similar to the postbuckling state of a column under an axial force. However, there is an important difference. For the column, the post-buckling state is maintained by the prescribed axial displacement at the two ends of the column. For the blanket film, no displacement is prescribed, so that the equilibrium wrinkles are unstable, and can reduce energy further by evolving into wrinkles of longer wavelengths. The wrinkles, however, coarsen slowly because of the viscosity of the BPSG layer. Consequently, the saturated wrinkles of any wavelength are in a kinetically constrained equilibrium state.

The mechanical property of the underlayer affects the wrinkles in the compressed film. This fact is brought to attention recently in the development of low dielectric constant polymers for interconnect structures ${ }^{[41]}$. These authors coat a silicon wafer with a polymer layer, and then deposit a SiN thin film on the polymer, with the SiN film under a compressive residual stress. The SiN film remains flat on annealing below the glass transition temperature $T_{\mathrm{g}}$ of the polymer underlayer, but wrinkles on annealing above the $T_{\mathrm{g}}$ of the underlayer. In both cases, wrinkles would lower the elastic energy stored in the SiN film. Below $T_{\mathrm{g}}$, the polymer underlayer is elastic, and the SiN film remains flat, because the wrinkles would add too much elastic energy to the underlayer. Above $T_{\mathrm{g}}$, the polymer underlayer creeps, and the SiN film wrinkles, the elastic energy in the underlayer decaying over a long time. Of course, if the polymer underlayer below $T_{\mathrm{g}}$ has a very low elastic modulus, the stressed film will form wrinkles, which only add a small amount of elastic energy in the underlayer. Such wrinkles are in a stable equilibrium state, and will not coarsen over time. This behavior is studied in a compressed gold film on a rubber ${ }^{[42]}$. The behaviors of the wrinkles, easily observable in experiments, provide a means to determine mechanical properties of the underlayer. 
We now analyze the equilibrium wrinkles, following a similar analysis for buckledelamination of thin films ${ }^{[43]}$. Consider a film that wrinkles in the $x_{1}$ direction, with nonzero displacements $w\left(x_{1}\right)$ and $u_{1}\left(x_{1}\right)$. With the general theory in Section 3 , we find that the membrane force relates to the displacements as

$$
N_{11}=-\frac{E h}{1-\nu} \varepsilon_{0}+\frac{E h}{1-\nu^{2}}\left[\frac{\mathrm{d} u_{1}}{\mathrm{~d} x_{1}}+\frac{1}{2}\left(\frac{\mathrm{d} w}{\mathrm{~d} x_{1}}\right)^{2}\right]
$$

In equilibrium, the tractions on the interface, $q$ and $T_{1}$, vanish, so that the moment balance Eq.(4) reduces to

$$
\frac{E h^{3}}{12\left(1-\nu^{2}\right)} \frac{\mathrm{d}^{4} w}{\mathrm{~d} x_{1}^{4}}=N_{11} \frac{\mathrm{d}^{2} w}{\mathrm{~d} x_{1}^{2}}
$$

When the shear traction $T_{1}$ vanishes, inplane force equilibrium requires that the membrane force $N_{11}$ be a constant in the film. Consequently, the ordinary differential Eq.(23) has constant coefficients. The nontrivial solution $w\left(x_{1}\right)$ is of the sinusoidal form of (14). Substituting (14) into (23), we obtain that

$$
N_{11}=-\left(\frac{\lambda_{\mathrm{c}}}{\lambda}\right)^{2} \frac{E h \varepsilon_{0}}{1-\nu}
$$

where $\lambda_{c}$ is the critical wavelength given by (18). The longer the wavelength, the lower the amplitude of the membrane force.

Substituting (14) and (24) into (22), we find the equilibrium wrinkle amplitude

$$
A_{\text {eq }}=\frac{h}{\sqrt{3}} \sqrt{\left(\lambda / \lambda_{\mathrm{c}}\right)^{2}-1}
$$

and the inplane displacement field

$$
u_{1}\left(x_{1}\right)=-\frac{\pi}{4 \lambda} A_{\text {eq }}^{2} \sin \left(\frac{4 \pi x_{1}}{\lambda}\right)
$$

In the equilibrium wrinkles, the inplane displacement undulates with the wavelength half that of the deflection.

For any wavelength $\lambda>\lambda_{\mathbf{c}}$, a nontrivial equilibrium state exists. Consequently, infinitely many such equilibrium states exist for a blanket film. On the other hand, the viscous layer controls the wrinkling rate. The fastest growing mode, of wavelength $\lambda_{\mathrm{m}}$, prevails at the initial stage. As the wrinkles approach the equilibrium state of wavelength $\lambda_{\mathrm{m}}$, creep slows down, and the film is kinetically constrained near this equilibrium state, making such state observable in experiment. Comparing (18) and (20), we note that $\lambda_{\mathrm{m}} / \lambda_{\mathrm{c}}=\sqrt{3}$. Consequently, the wrinkles of wavelength $\lambda_{\mathrm{m}}$ have equilibrium amplitude $A_{\mathrm{eq}}=\sqrt{2 / 3} \mathrm{~h}$. This agrees reasonably well with the experiment (Fig.7).

We next consider fracture induced by the wrinkles. At the crest of a wrinkle, the stress is the sum of that due to the membrane force, and that due to the bending moment, namely

$$
\sigma=\frac{N_{11}}{h}-\frac{E h}{2\left(1-\nu^{2}\right)} \frac{\mathrm{d}^{2} w}{\mathrm{~d} x_{1}^{2}}
$$

For equilibrium wrinkles of wavelength $\lambda$, this stress is

$$
\sigma_{\text {eq }}=\frac{E \varepsilon_{0}}{1-\nu}\left(\frac{\lambda_{\mathrm{c}}}{\lambda}\right)^{2}\left[2 \sqrt{3\left(\left(\lambda / \lambda_{\mathrm{c}}\right)^{2}-1\right)}-1\right]
$$


The stress becomes tensile when $\lambda / \lambda_{\mathrm{c}}>\sqrt{13 / 12}$. For wrinkles of the wavelength $\lambda_{\mathrm{m}}$, Eq. (28) simplifies to $\sigma_{\text {eq }} \approx 1.3 \varepsilon_{0} E /(1-\nu)$. Using representative values, $\varepsilon_{0}=0.012, E=150 \mathrm{GPa}$ and $\nu=0.3$, we find that $\sigma_{\mathrm{eq}}=3.3 \mathrm{GPa}$. The toughness of $\mathrm{SiGe}$ is about $K_{\mathrm{c}} \approx 1 \mathrm{MPa} \sqrt{\mathrm{m}}$. The critical flaw size is $a_{\mathrm{c}} \approx\left(K_{\mathrm{c}} / \sigma_{\mathrm{eq}}\right)^{2} / \pi \approx 29 \mathrm{~nm}$, which is close to the thickness of the SiGe films. Flaws of this size are expected at the edges of the islands.

\section{THE RACE BETWEEN EXPANSION AND WRINKLING}

The shear lag model describes the island expansion, neglecting wrinkling. The two wrinkling models analyze a blanket film, neglecting the island expansion. In reality, an island expands and wrinkles concomitantly. We adopt the Reynolds lubrication theory ${ }^{[44]}$ to model the creep in the BPSG layer, taking into account the boundary conditions imposed by the elastic island. The velocity field relates to the tractions as ${ }^{[30]}$

$$
\begin{aligned}
& \frac{\partial u_{\alpha}}{\partial t}=\frac{H^{2}}{2 \eta} \frac{\partial q}{\partial x_{\alpha}}+\frac{H}{\eta} T_{\alpha} \\
& \frac{\partial w}{\partial t}=\frac{\partial}{\partial x_{\alpha}}\left(-\frac{H^{3}}{3 \eta} \frac{\partial q}{\partial x_{\alpha}}-\frac{H^{2}}{2 \eta} T_{\alpha}\right)
\end{aligned}
$$

where $H=H_{0}+w\left(x_{1}, x_{2}, t\right)$, with $H_{0}$ being the average thickness of the glass layer. The lubrication theory describes the three dimensional flow in the glass layer with partial differential equations in two dimensions, and is justified when the thickness of the glass layer is small compared to the wrinkle wavelength. The shear lag model is a special case of the lubrication theory, as is evident by comparing (11) and (29).

Recall that the tractions relate to the displacement field by the elastic plate theory, which, together with (29) and (30), governs the displacement field. Figure 8 shows the computed wrinkle amplitude at the island center as a function of time ${ }^{[32]}$. Included are islands of three sizes, $L=15 \mu \mathrm{m}, 30 \mu \mathrm{m}, 60 \mu \mathrm{m}$. The dashed lines are the results of linear

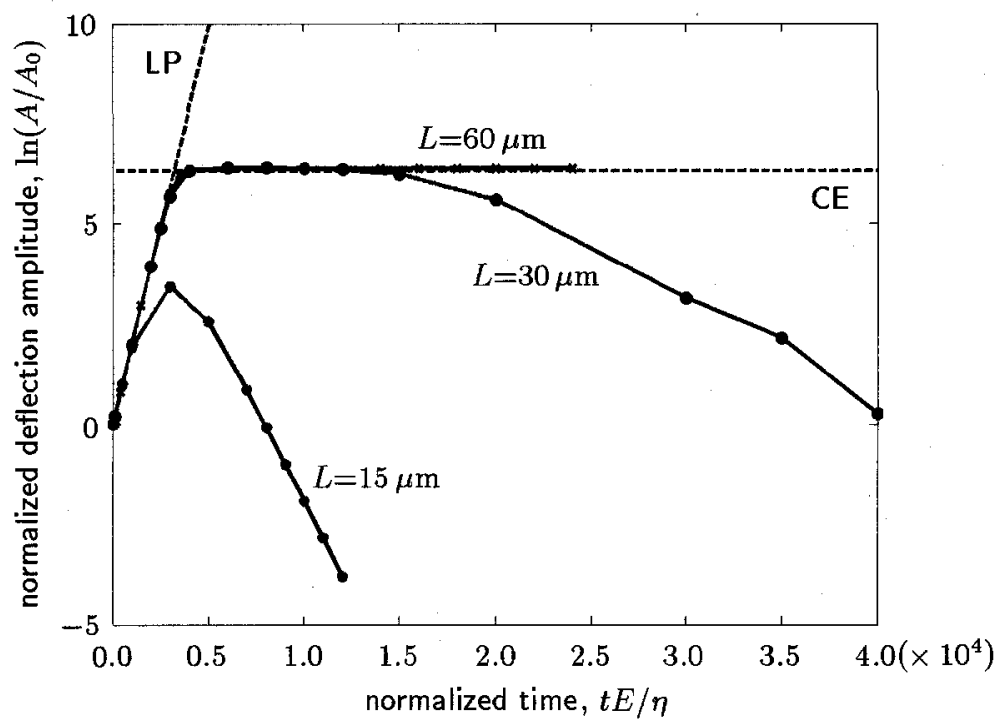

Fig.8 The wrinkle amplitude as a function of time for various islands sizes from numerical simulations ${ }^{[32]}$ 
perturbation (LP) and constrained equilibrium (CE). The wrinkle amplitude of every island grows exponentially initially, as predicted by the linear perturbation analysis. For the island of size $L=15 \mu \mathrm{m}$, the expansion reaches the island center before the wrinkle amplitude attains CE and, subsequently, the wrinkle amplitude decays. For a larger island ( $L=$ $30 \mu \mathrm{m}$ ), the wrinkle amplitude attains CE, stays there for a while, and then decays when the expansion arrives. For a still larger island $(L=60 \mu \mathrm{m})$, the wrinkle amplitude stays at $\mathrm{CE}$ for a longer time, but eventually will also decay when the expansion arrives.

Experimental observations have indicated that, with everything else being set the same, there exists a critical island size, $L_{\mathrm{c}}$ : smaller islands expand, and larger islands wrinkle and fracture. What determines the critical island size? Clearly the wrinkle wavelength does not set the critical island size. As shown in Fig.2, the wrinkle wavelength is about $1 \mu \mathrm{m}$, and our experiment shows that the critical island size is somewhere between $30 \mu \mathrm{m}$ and $80 \mu \mathrm{m}$. It is the race between expansion and wrinkling that determines the critical island size. The expansion time given by Eq.(13) depends on the film size $L$, but the wrinkling time given by Eq.(21) does not. For a small island, the expansion reaches the island center in a short time, before the wrinkles grow much. For a large island, the expansion takes a long time to reach the island center, so that the wrinkles have a plenty of time to amplify to cause fracture. The critical island size is estimated by setting $t_{\mathrm{E}}=t_{\mathrm{W}}$, giving

$$
L_{\mathrm{c}}=\frac{\pi}{\left[(1+\nu) \varepsilon_{0}\right]^{3 / 4}} \sqrt{\frac{3 H h}{2} \ln \left(\frac{A_{\mathrm{c}}}{A_{0}}\right)}
$$

where $A_{\mathrm{c}}$ is a critical wrinkle amplitude, at which the tensile stress at the wrinkle crests may cause fracture. Although the value $A_{\mathrm{c}}$ depends on the flaw size in the film, the critical island size $L_{\mathrm{c}}$ only weakly depends on $A_{\mathrm{c}}$. An estimate from Eq.(31) gives $L_{\mathrm{c}} \approx 20 \mu \mathrm{m}$, which agrees with the experimental observation reasonably well. The critical island size is independent of the glass viscosity $\eta$, because reducing the viscosity accelerates the expansion and wrinkling proportionally.

\section{WRINKLE SUPPRESSION BY CAP LAYER}

Figure 9 illustrates a process that can fabricate islands larger than the critical island size described above. Before annealing, a strain-free cap layer is deposited on the strained $\mathrm{SiGe}$ island. After first annealing, the cap-SiGe bilayer reaches the force balance, tensile in the cap layer, and compressive in the SiGe island. The compressive stress in $\mathrm{SiGe}$ is reduced from the initial state. Then, the cap layer is removed and the remaining SiGe film is annealed again to achieve a full relaxation. During the first annealing, since the effective thickness of the island is larger and the effective compressive stress is smaller, the critical island size is larger than the original critical island size for SiGe islands without the cap layer. In the second annealing, since the compressive strain in SiGe has been partially relaxed, the critical island size is also larger.

Figure 10 shows the micrographs of several annealed islands $(L=200 \mu \mathrm{m}$ and $500 \mu \mathrm{m})$. Without the cap, the islands of both sizes fracture. With a $19 \mathrm{~nm}$ thick cap, the $200 \mu \mathrm{m}$ island is crack-free, but the $500 \mu \mathrm{m}$ island still factures although the improvement is obvious. A thicker cap is required to relax the $500 \mu \mathrm{m}$ islands crack-free. For very large islands, multiple etching and annealing steps may be required. 


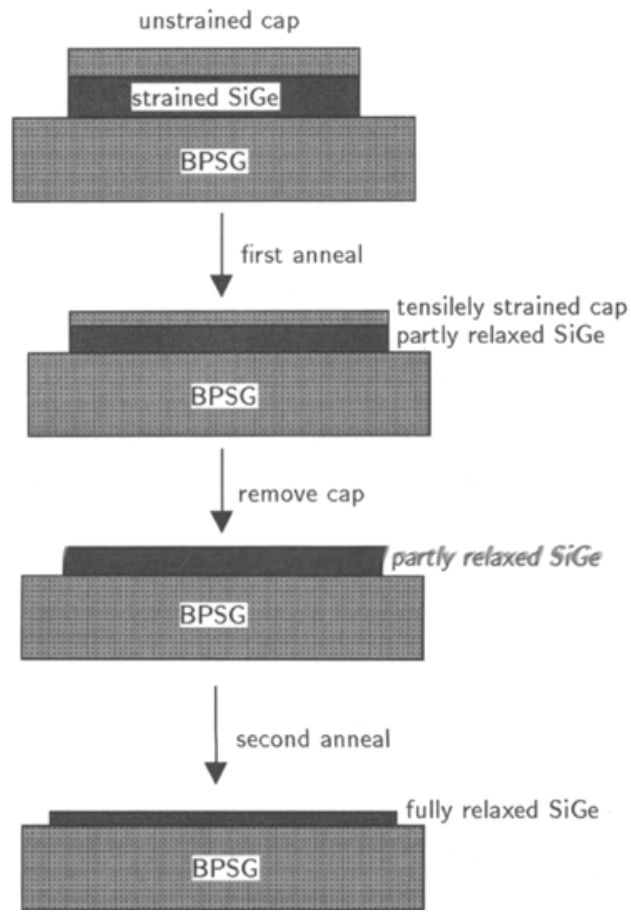

Fig.9 Schematic of the two-step annealing

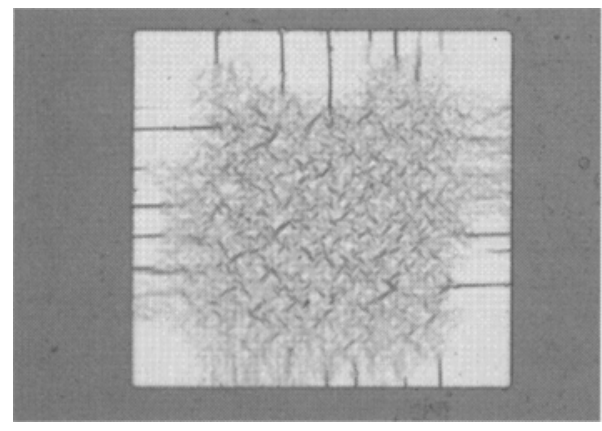

(a) $L=200 \mu \mathrm{m}$, without cap

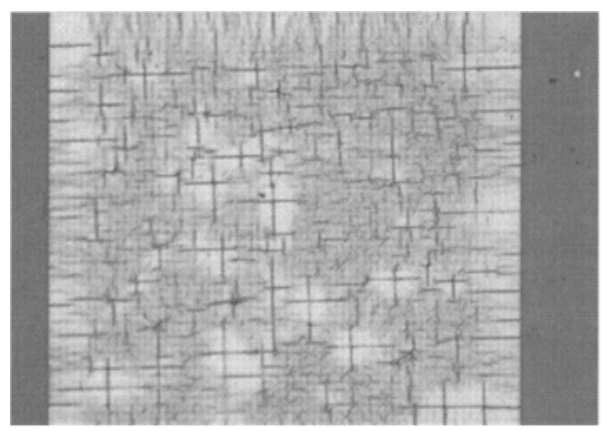

(c) $L=500 \mu \mathrm{m}$, without cap

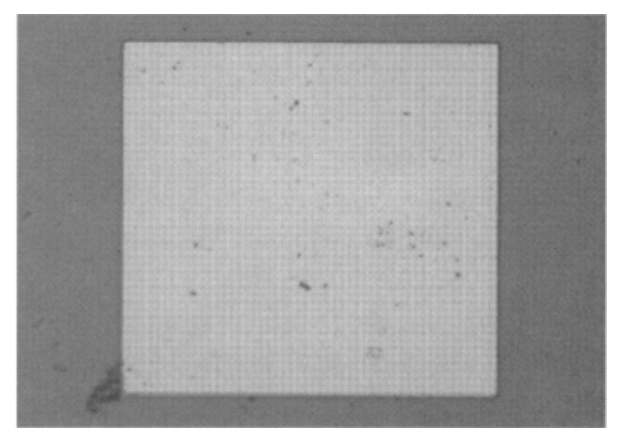

(b) $L=200 \mu \mathrm{m}$, with a $19 \mathrm{~nm}$ thick cap

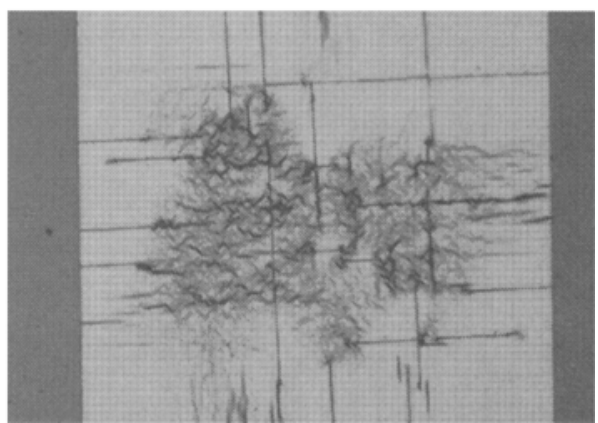

(d) $L=500 \mu \mathrm{m}$, with a $19 \mathrm{~nm}$ thick cap

Fig:10 Large islands annealed with and without cap 


\section{CONCLUDING REMARKS}

This paper describes a series of studies on a compressed SiGe island on a glass layer. Annealing is in effect a race between two processes: expansion and wrinkling. For a small island, expansion prevails, and the island relaxes to the stress-free state. For a large island, wrinkling prevails, and the tensile stress develops and the island fractures. We model the island as an elastic plate, and the glass layer as a viscous liquid. The theory is compared with the experiment at various points. Using a cap layer, we can relax large islands and avert wrinkle fracture. The behaviors of thin film wrinkles, so easily observable in experiments, provide a means to measure mechanical properties of the underlayer. For example, our experimental data of the wrinkle amplitude as a function of time provide a reliable means to determine the viscosity of BPSG.

\section{REFERENCES}

1 Freund LB. The mechanics of electronic materials. Int $J$ Solids Structures, 2000, 37(1 2): $185 \sim 196$

2 Suo Z. Evolving material structures of small feature sizes. Int J Solids Structures, 2000, 37(1 2): $367 \sim 378$

3 Gao H, Nix WD. Surface roughening of heteroepitaxial thin films. Ann Rev Mater Sci, 1999, 29: $173 \sim 209$

4 Bruner K. Si/Ge nanostructures. Rep Porg Phys, 2002, 65(1): 27 72

5 Tong QY, Gosele U. Semiconductor Wafer Bonding: Science and Technology. New York: Wiley, 1998

$6 \mathrm{Yu} \mathrm{H}$, Suo Z. A model of wafer bonding by elastic accommodation. J Mech Phys Solids, 1998, 46(5): $829 \sim 844$

7 Li M, Wang J, Zhuang L, et al. Fabrication of circular optical structures with a $20 \mathrm{~nm}$ minimum feature size using nanoimprint lithography. Appl Phys Lett, 2000, 76(6): 673 675

$8 \mathrm{Kim}$ C, Burrows PE, Forrest SR. Micropatterning of organic electronic devices by cold welding. Science, 2000, 288(5467): 831 833

9 Josell D, Wheeler D, Huber WH, et al. Superconformal electrodeposition in submicron features. Phys Rev Lett, 2001, 87(1): 016102

10 Liang J, Suo Z. Stable island arrays by height-constrained Stranski-Krastanov growth. Applied Physics Letters, 2001, 79(20): 3251 3252

11 Suo Z, Lu W. Forces that drive self-assembly on solid surfaces. Journal of Nanoparticles Research, 2000, 2(4): 333 344

12 Chou SY, Zhuang L, Gao L. Lithographically induced self-construction of polymer microstructures for resistless patterning. Appl Phys Lett, 1999, 75(7): 1004 1006

13 Erlebacher J, Aziz MJ, Chason E, et al. Nonlinear amplitude evolution during spontaneous patterning of ion-bombarded $\mathrm{Si}(001)$. J Vac Sci Technol, A, 2000, 18(1): 115 120

14 Ismail K, Arafa M, Saenger KL, et al. Extremely high electron mobility in Si/SiGe modulationdoped heterostructures. Appl Phys Lett, 1995, 66(9): 1077 1079

15 Xie YH, Monroe D, Fitzgerald EA, et al. Very high mobility two-dimensional hole gas in $\mathrm{Si} / \mathrm{Ge}_{x} \mathrm{Si}_{1-x} / \mathrm{Ge}$ structures grown by molecular beam epitaxy. Appl Phys Lett, 1993, 63(16): $2263 \sim 2265$

16 Mooney PM, Chu JO. SiGe technology: heteroepitaxy and high-speed microelectronics. Annu Rev Mater Sci, 2000, 30: 335 362

17 Fitzgerald EA, Xie YH, Monroe D, et al. Relaxed $\mathrm{Ge}_{x} \mathrm{Si}_{1-x}$ structures for III-V integration with $\mathrm{Si}$ and high mobility two-dimensional electron gases in Si. J Vac Sci Technol, B, 1992, 
10(4): $1807 \sim 1819$

18 Fitzgerald EA, Xie YH, Green ML, et al. Totally relaxed $\mathrm{Ge}_{x} \mathrm{Si}_{1-x}$ layers with low threading dislocation densities grown on Si substrates. Appl Phys Lett, 1991, 59(7): 811 813

19 Fitzgerald EA. GeSi/Si nanostrcutures. Annu Rev Mater Sci, 1995, 25: 417 454

20 Lo YH. New approach to grow pseudomorphic structures over the critical thickness. Appl Phys Lett, 1991, 59(18): 2311 2313

21 Powell AR, Iyer SS, LeGoues FK. New approach to the growth of low dislocation relaxed SiGe material. Appl Phys Lett, 1994, 64(14): 1856 1858

22 Yang $\mathrm{Z}$, Alperin J, Wang WI, et al. In situ relaxed $\mathrm{Si}_{1-x} \mathrm{Ge}_{x}$ epitaxial layers with low threading dislocation densities grown on compliant Si-on-insulator substrates. $J$ Vac Sci Technol, B, 1998, 16(3): 1489 1491

23 Huang FY, Chu MA, Tanner MO, et al. High-quality strain-relaxed SiGe alloy grown on implanted silicon-on-insulator substrate. Appl Phys Lett, 2000, 76(19): 2680 2682

24 Vanhollebeke K, Moerman I, Van Daele P, et al. Compliant substrate technology: investigation of mismatched materials for opto-electronic applications. Progress in Crystal Growth and Characterization of Materials, 2000. 1 55

25 Hobart KD, Kub FJ, Fatemi M, et al. Compliant substrates: a comparative study of the relaxation mechanisms of strained films bonded to high and low viscosity oxides. J Electronic Materials, 2000, 29(7): 897 900

26 Movan PD, Kuech TF. Kinetics of strain relaxation in semiconductor films grown on borosilicate glass-bonded substrates. J Electronic Materials, 2001, 30(7): 802 806

27 Huang R, Yin H, Liang J, et al. Relaxation of a strained elastic film on a viscous layer. Mater Res Soc Symp Proc, 2001, 695: 115 120

28 Sridhar N, Srolovitz DJ, Suo Z. Kinetics of buckling of a compressed film on a viscous substrate. Appl Phys Lett, 2001, 78(17): 2482 2484

29 Huang $\mathrm{R}$, Suo Z. Instability of a compressed elastic film on a viscous layer. Int $J$ Solids Struct, 2002, 39(7): 1791 1802

30 Huang R, Suo Z. Wrinkling of a compressed elastic film on a viscous layer. J Appl Phys, 2002, 91(3): 1135 1142

31 Sridhar N, Srolovitz DJ, Cox BN. Buckling and post-buckling kinetics of compressed thin films on viscous substrates. Acta Mater., 2002, 50(10): 2547 2557

32 Liang J, Huang R, Yin H, et al. Relaxation of compressed elastic islands on a viscous layer. Acta Materialia, 2002, 50(11): 2933 2944

33 Yin H, Huang R, Hobart KD, et al. Strain relaxation of SiGe islands on compliant oxide. $J$ Appl Phys, 2002, 91(12): 9716 9722

34 Timoshenko S, Woinowsky-Krieger S. Theory of Plates and Shells, 2nd edition. New York: McGraw-Hill, Inc, 1987

35 Landau D, Lifshitz EM. Theory of Elasticity. London: Pergamon Press, 1959

36 Bachelor GK. An Introduction to Fluid Dynamics. Cambridge: Cambridge University Press, 1967

37 Elsasser WM. Convection and stress propagation in the upper mantle. In: Runcorn WK ed. The Application of Modern Physics to the Earth and Planetary Interiors, New York: Weily, 1969. 223 246

38 Bott MH, Dean DS. Stress diffusion from plate boundaries. Nature, 1973, 243: 339 341

39 Lehner FK, Li VC, Rice JR. Stress diffusion along rupturing plate boundaries. J Geophysical Research, 1981, 86(B7): 6155 6169

40 Suo $\mathrm{Z}$. Wrinkling of the oxide scale on an aluminum-containing alloy at high temperatures. $J$ Mech Phys Solids, 1995, 43(6): 829 846 
41 Martin SJ, Godschalx JP, Mills ME, et al. Development of a low-dielectric-constant polymer for the fabrication of integrated circuit interconnect. Advanced Materials, 2000, 12(23): 1769 1778

42 Bowden N, Brittan S, Evans AG, et al. Spontaneous formation of ordered structures in thin films of metals supported on an elastomeric polymer. Nature, 1998, 393(6681): 146 149

43 Hutchinson JW, Suo Z. Mixed-mode cracking in layered materials. Advances in Applied Mechanics, 1991, 29: 63 191

44 Reynolds $\mathrm{O}$. On the theory of lubrication and its application to Mr. Beauchamp Tower's experiments, including an experimental determination of the viscosity of olive oil. Phil Trans Roy Soc, London, 1886, 177: 157 234 